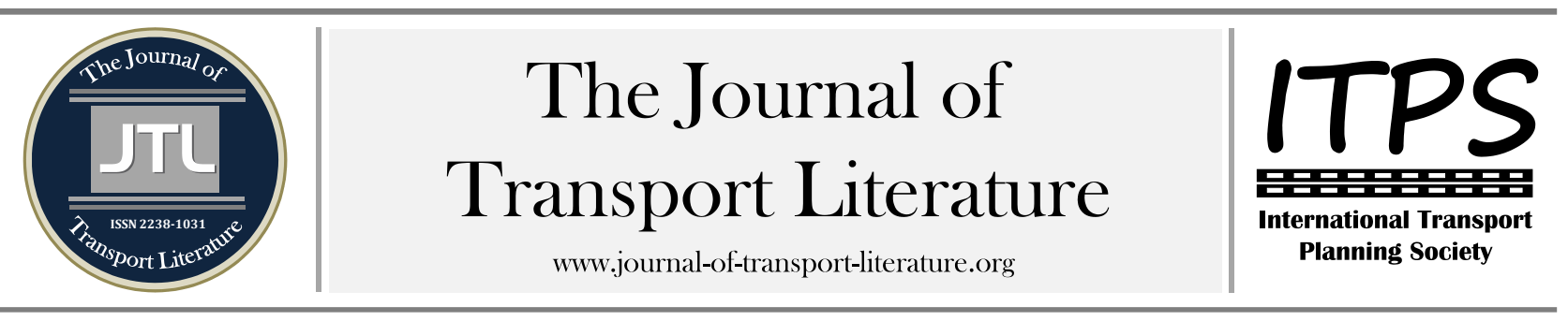

\title{
An investigation of factors influencing performance of Radio Frequency Identification (RFID): applications in transportation
}

\author{
Don Nash II ${ }^{1}$; Judith L. Mwakalonge ${ }^{2,+}$; Judy A. Perkins ${ }^{1}$ \\ ${ }^{1}$ Civil and Environmental Engineering Prairie View A \& M University, Prairie View, United States \\ 2 Civil and Mechanical Engineering Technology, Orangeburg, United States
}

\begin{tabular}{l} 
Article Info \\
\hline Keywords: \\
RFID \\
Speed \\
Reader \\
Tag \\
Submitted 22 Apr 2013; \\
received in revised form 24 Apr 2015; \\
accepted 12 Jul 2015. \\
Licensed under \\
Creative Commons \\
CC-BY 3.0 BR.
\end{tabular}

\begin{abstract}
This study investigates the effect of speed, tag and reader location, vehicle speed, and over-shadowing on RFID performance. The study tested two reader/antenna heights and three tag locations. Three vehicle speeds were tested for each tag location including the simultaneous placement of RFID tags on the vehicle. The study results show, with tags individually placed on the vehicle, the appropriate position for a tag is on the vehicle windshield, and the reader/antenna set at $7 \mathrm{ft}$ above the ground on a signpost. Placing multiple tags on the vehicle simultaneously improved the signal strength and detection rates. Vehicle speed had a negative effect on tag detection rate; that is, at higher vehicle speeds the detection rate decreased. With respect to horizontal distance between the reader and the tag, the results show that, the closer the tag and reader are to each other the higher the signal strength and so is the detection rate.
\end{abstract}

+ Corresponding author. South Carolina State University. 300 College Street NE P.0. Box 8144, South Carolina 29118.

E-mail address: jmwakalo@scsu.edu.

\section{Introduction}

The Radio Frequency Identification (RFID) technology uses radio waves to exchange information between a reader and an electronic tag attached to an object for the purpose of identification and tracking. The application of the technology can be traced back to World War II when it was used to distinguish enemy warplanes from allied warplanes (Roberti, 2012). The growth of computing capabilities and the low cost of this technology has fueled its application in different disciplines for asset tracking, highway toll collection, key chain devices to open car doors, track population of wild animals, and hospital operating rooms. In the transportation industry, RFID has been used since the mid-1980s (The basics of RFID, 2011) with tags attached to chassis carriers to serve as "license plates" and for toll collections on toll roads. At toll facilities RFID, as part of the electronic toll collection system, has been proven to process as many as 2,200 cars per hour compared to only 400 cars per hour by a manual tollbooth (Mwape, 2009). The great success of RFID technology in toll collection has prompted the investigation of its applicability into other areas of the transportation sector both in the US and abroad.

In 2004, the Orlando/Orange County Expressway Authority (OOCEA), started deploying an RFID-based trafficmonitoring system in central Florida. The pilot study covered 228 miles of toll and non-toll state highways on which RFID readers were mounted on the roadside to collect signals from transponders already installed in about 1 million E-Pass and SunPass customer vehicles. The goal was to implement a system that would trace the travel time of individual cars as they passed the roadside readers, create an average travel time, and then disseminate that information to the public. Currently, the agency distributes mini-transponders free (OOCEA, 2011). In recent years, more agencies including Houston Transtar (2011) are using toll tags in combination with other methods to collect travel time and speed for traffic monitoring purposes. In 2008 the Bicycle Commuter Act, allowed employers to give employees who commute by bike a monthly taxfree stipend of up to $\$ 20$ as a way to promote biking. Seeing the opportunity to provide employers with an accurate count of biking employees, the Dero Bike Rack Company developed an RFID bicycle counting system. The RFID readers were installed in select exterior locations around a business or on campus to provide a "ZAP Zone" that is the bicycle detection zone. Bicycle commuters with bicycle-mounted passive RFID tags entering the "ZAP Zone" were then accurately logged and counted for reimbursement purposes. The system was released on January 1, 2010, and as of March 17, 2010, the company counted 2,006 bike commutes and 10,421 bicycle miles (Stratos, 2011).

In 2010, Utah Department of Transportation (UDOT) launched a time variant and demand responsive electronic tollcollection system on a high occupancy vehicle (HOV) and high occupancy toll (HOT) lane on I-15. The system uses EPC Gen 2 passive ultrahigh frequency (UHF) RFID tags. The tags are capable of transmitting their ID numbers to readers located 20 feet or more away from vehicles traveling at a speed of 55 miles per hour or higher. The user attaches the transponder onto the vehicle windshield and readers mounted above the highway capture tag ID numbers, enabling UDOT to charge each driver a varying amount of money based on the zone in which he or she drives, as well as the time of day and the 
amount of traffic present at that time. The tag has an on-off switch that enables the user to activate or deactivate the tag. When it is in the off mode, a red line appears on both the front and back of the tag, enabling the police to visually determine whether the transponder is activated when the driver has no passengers and thus pay for using the HOV lane.

Researchers at Virginia Tech, (Yates, 2009; Fedrowitz, 2007) investigated the use of RFID for Virginia Department of Transportation to manage highway assets located in the right of way. In these research studies, static and dynamic tests were carried out. For static testing, researchers tested the effect of horizontal distance between the tag mounted on a metal mile marker and a hand held mobile reader. For dynamic testing, the studies investigated the effect of vehicle speed and horizontal distance between the tag mounted on a metal mile marker and a reader mounted on a vehicle. The horizontal distance tested included 5, 10, 25, 50, and 100 feet from the tag. The researchers also recorded the maximum distance a tag can be detected by a reader. Vehicle speed tested included 10,20,30, and 60 miles per hour. The study found that the long range system read up to 115 feet from a tag mounted to a mile marker sign under static conditions (vehicle not moving). In addition, the maximum dynamic read range of a vehicle traveling at $10 \mathrm{mph}$ was also 115 feet. At a highway speed of 60 to $65 \mathrm{mph}$, the dynamic system was not very consistent but was capable of reading a tag at a maximum distance of 25 feet.

Ross et al. (2009) tested the applicability of RFID to track the progress of construction materials being tested by the Georgia Department of Transportation (GDOT) Office of Materials and Research (OMR). The study used both mobile and fixed readers to identify and track applied tags under extreme laboratory testing conditions including moisture, $\mathrm{pH}$, temperature, and pressure. The study found that RFID tags are durable enough to be used to track the progress of construction materials and to locate misplaced samples in the laboratory.

In summary, most applications of RFID in transportation include toll collection and traffic management. In these applications, the tag is usually located on the vehicle windshield and license plates. However, past studies indicated that the location of the tag on a vehicle can affect performance of RFID technology. For example, North Texas Toll Authority (NTTA, 2011) identified some vehicle features that affect RFID tag performance as being windshield mirror with lane departure warning system, heated windshield, insulated or insta-clear glass, and solar control. Additionally, RFID specifications indicated that the technology performed better when the tags or readers are not located low to the ground (RF Code, 2012). This indicates that tag locations on the vehicle have influence on RFID performance. Additionally, a literature review revealed that most readers have an anti-collision system that allows readers to read multiple tags simultaneously. This may result in a tag over-shadowing effect, particularly in situations where vehicles with RFID tag are close to each other such as in a traffic queue or at toll facilities. Understanding such effect is important for further implementation of this technology in transportation related applications.

Based on the foregoing discussion, this paper has two specific objectives. First, investigate the performance of RFID technology by varying (1) tag locations on the vehicle, (2) reader mounting locations on a signpost, (3) vehicle speed, and (4) horizontal distance between the tag and reader; and, second, investigate the effect of tag over-shadowing on RFID performance.

The remainder of the paper is organized as follows. In Section 2, the details of RFID technology employed in this study are presented. Section 3 first discusses the site characteristics, and then presents the field testing or data collection. The first part of the results subsection reports on different scenarios that encompass the combination of tag location, reader location from the ground, and whether tags were simultaneous or not. The second part of the results-subsection reports on the regression analysis. Finally, highlights on what has been learned from this experimental study on the effect of tag and reader location, driving speed, and over-shadowing effects on performance of RFID technology is presented on conclusions and recommendations for future research section.

\section{RFID Technology}

RFID technology uses radio waves to exchange information between the transponder and the reader. Depending on mobility, RFID readers are classified as fixed or mobile. Fixed readers detect tag activity while stationary and typically have higher a reading range whereas mobile readers sense tag availability while mounted on a vehicle or any moving object. On the other hand, RFID tags are classified based on the way they receive power. Active tags are battery powered, semi-active tags are semi-battery powered, and passive tags are non-battery powered.

The choice of tag for a particular application depends mainly on its cost, reliability, and capability. Passive tags are cheaper compared to semi-active and active tags. Typically, information exchange between active tags to active readers is much more reliable than from passive tags. Many active tags today have operational ranges of hundreds of meters, and a battery life of up to 10 years (Lodgher et al, 2010). Further, tags are classified as read only or read-write. The former are programmed with a unique ID that can never be changed and read-write allows the user to add or write over existing information when the tag is within range of the reader. This study used active RFID read only tags because of their high reading range and a fixed reader manufactured by RF CodeTM.

\section{Site Characteristics}

The factors used for selecting a suitable site for testing to achieve the objectives of the study were geometric characteristics, posted speed limit, traffic volume, and presence of metal traffic signs. In this study, a roadway with a speed limit of $55 \mathrm{mph}$, low traffic volume, and metal traffic signs was selected for conducting the test run demonstrations. This roadway was selected because it has low traffic volume and favorable roadway geometric characteristics that allows for high-speed dynamic testing. Further, the site has driveways in its vicinity to facilitate turning of the test vehicle once it has passed the interrogation zone. The site is located along Farm to Market 1098 (FM 1098) running in a north-south direction very close to Prairie View A\&M University main campus. Although freeways offer high speed, they were excluded from consideration due to their limited access and high traffic volume. The selected site has the following characteristics: twoway highway with $12 \mathrm{ft}$ lanes and $6 \mathrm{ft}$ shoulder; traffic sign is $9 \mathrm{ft}$ from the shoulder and $20 \mathrm{ft}$ from the center of the road; posted speed limit is $55 \mathrm{mph}$; and the traffic sign is $8 \mathrm{ft}$ high. 


\section{Field Testing}

The process of field testing was carried out after determining data needs, creating data collection protocol, and identifying equipment needed. The main materials needed for the testing were the reader, laptop, power source, RFID tags, and adhesive. To test a wide range of scenarios, the study considered several variables that may affect the readability of the RFID tag. The variables included the height of the reader/antenna, speeds of the vehicle, and placement of the tag on the vehicle. The tag locations tested were the windshield, door handle, and license plate, which were about 5.5, 3.42, and $1.33 \mathrm{ft}$ respectively, above ground for a DODGE pickup truck as shown. The windshield and license plate are locations that are widely used in practice to attach tags. For the reader placement on a traffic sign, scenarios tested included setting the reader at a height of about 4 feet and 7 feet above ground level. The two heights where chosen to represent different types of traffic control devices that are in use such as delineators, mile markers, speed limit signs, etc. In addition, heights lower than 4 feet were not considered because manufacturer specifications for the M200 indicated that the reader would not properly detect tag activity if the reader/antenna was low to the ground. The vehicle speeds tested included 5, 10, 15, 20, 25, and 35 mph. At $35 \mathrm{mph}$, the detection rate was very low, and therefore, higher speeds were not tested. For each height of tag and reader placement, three (3) different speeds were tested each for 10 runs.

A vehicle with a tag attached on it on either or all of the three locations (door handle, front license plate, and windshield) passed near the reader/antenna at a selected testing speed and a computer connected to the reader recorded whether the tag was detected and signal strength (received signal strength indicator, RSSI) for each detection. This step was repeated 10 times on a two-lane road in each direction to examine the effect of horizontal distance on detection rate and signal strength.

\section{Data Analysis}

Scenario 1: Reader Height 4ft and Non Simultaneous Tags. Figure 1 presents the tag readability results for scenario 1. The chart shows a clear relationship between the selected variables and readability of the tags. Each tag position had 60 runs in total resulting from ten trials, three different speeds, and each driving direction, that was north and south bound. As observed from the figure, with respect to tag location, the windshield had higher reading rates of 66.7 percent followed by the door handle at 25 percent, and lastly, the license plate at 6.7 percent. For windshield, the detection rate is higher at low speeds and lower at high speeds. At $15 \mathrm{mph}$, the detection rate was 90 percent whereas at $30 \mathrm{mph}$ the detection rate was 50 percent. A similar trend was observed for the license plate; at $15 \mathrm{mph}$ the detection rate was 40 percent and 15 percent at $30 \mathrm{mph}$. The detection rate for a tag on the license plate showed an unexpected trend. The tag was detected at $10 \mathrm{mph}$ with a 15 percent detection rate but none at $15 \mathrm{mph}$ and a 5 percent detection rate at $20 \mathrm{mph}$. Reasons for this trend are unclear and therefore further research is required. In general, with respect to speed, detection rate decreases with the increase in speed and this is consistent with results of other studies (Yates, 2009; Fedrowitz, 2007). With regard to signal strength, windshield and door handle yielded the highest values compared to the license plate and this was in line with the readability trend.

Scenario 2: Reader Height $\mathbf{7 f t}$ and Non Simultaneous Tags. Figure 2 depicts the results for the reader mounted at 7 feet above the ground on a metal traffic sign. As shown in Figure 2, the detection rate was higher with the RFID tag on the windshield and lower with the RFID tag on the license plate. The detection rate with a tag on the windshield was 83.3 percent, which was higher by 16.6 percent compared to that of the reader mounted at 4 feet as presented in Figure 1 . Similar results occurred for a tag on the door handle and license plate. The detection rates for door handle and license plate were 30.7 percent and 6.6 percent higher than those reported in Figure 1. This implies that reader location has an effect on detection rates and the higher the better. With a tag on the windshield, at $15 \mathrm{mph}$, the detection rate was 95 percent, 85 percent at $25 \mathrm{mph}$, and 70 percent at $35 \mathrm{mph}$. This trend is consistent with expectations because at a lower speed, vehicles spend more time in the detection zone and therefore have a high chance for detection. The detection rates for tags on the door handle or license plate at different speeds showed an unexpected trend. With the tag on the door handle, the detection rate was higher for $35 \mathrm{mph}$ compared to $25 \mathrm{mph}$ and with the tag on the license plate, the detection rate was 5 percent more at $20 \mathrm{mph}$ compared to $10 \mathrm{mph}$. Other factors than those considered in the study may have influence on the performance of RFID technology and this should be investigated in future studies. With regard to signal strength, the windshield and door handle yielded the highest values compared to the license plate and this in line with detection rate trends. The 15 mph is common for all tag and reader locations. A further analysis was conducted and results presented in Figure 3. As shown in the figure, detection rates increased from license plate (LP) to door handle (DH) to windshield (WS) and are higher for readers at 7 feet compared to readers at 4 feet. Similarly, for readers at 7 feet, the signal strength (RSSI) values increased from license plate (LP) to door handle (DH) to windshield (WS). However, with a reader at 4 feet, there is a marginal difference between RSSI values for DH and WS. At 4 feet, no tag was detected for the license plate and therefore no detection rate or RSSI value is reported on the figure.

Scenario 3: Reader Height $4 \mathrm{ft}$ and Simultaneous Tags. Figure 4 presents the results for the reader set at 4 feet above the ground and the tags set simultaneously on the vehicle on three locations. This was done to investigate the effect of overshadowing on RFID performance. From the figure, it was observed that the detection rate followed the same trend as that of Scenario 1, but with higher detection rates for windshield and door handle. With simultaneous tags, the tag on the license plate was not detected. Similar to results presented in Scenario 1, with the tag on the windshield, the increase in vehicle speed corresponded to a decrease in detection rate. The tag on the door handle yielded unexpected results, with detection rates of 42 percent at $25 \mathrm{mph}$ and 45 percent at $35 \mathrm{mph}$. The tag on the windshield yielded a higher signal strength value compared to the tag on the door handle. This may be attributed to differences in tag height. Comparing this performance with the non-simultaneous performance presented in Scenario 1, it is evident that having simultaneous tags yields better performance for the door handle and windshield but not for the license plate.

Scenario 4: Reader Height $\mathbf{7 f t}$ and Simultaneous Tags. Figure 5 presents the results for the reader mounted at 7 feet above the ground on a metal traffic sign while tags are located simultaneously on the vehicle. As observed, the results show 
that the detection rates are higher with tags on the windshield compared to having tags on the door handle or license plate. Further, it was observed that the increase in vehicle speeds had a negative impact on tag readability. The signal strength is higher for tags on the windshield followed by tags on door handle and lastly with tags on the license plate. Comparing simultaneous and non-simultaneous with the reader/antenna at $7 \mathrm{ft}$, the detection rates were higher for simultaneous tags compared to non-simultaneous tags. The reason behind this observation needs further research. The $15 \mathrm{mph}$ is common for all reader locations and is used to further investigate the effect of other variables at a constant speed. Figure 6 presents the results of this analysis. As observed from the figure, the detection rates of 100 percent were achieved with the reader at 7 feet for tags on the door handle (DH) and the windshield (WS). With the reader at 4 feet, the detection rate increased from DH to WS. Similarly, for a reader at 7 feet, the signal strength (RSSI) values increased from license plate (LP) to door handle (DH) to windshield (WS). With a reader at 4 feet, the RSSI values increased from DH to WS. At 4 feet, no tag was detected for the license plate and therefore no detection rate or RSSI value was reported on the figure.

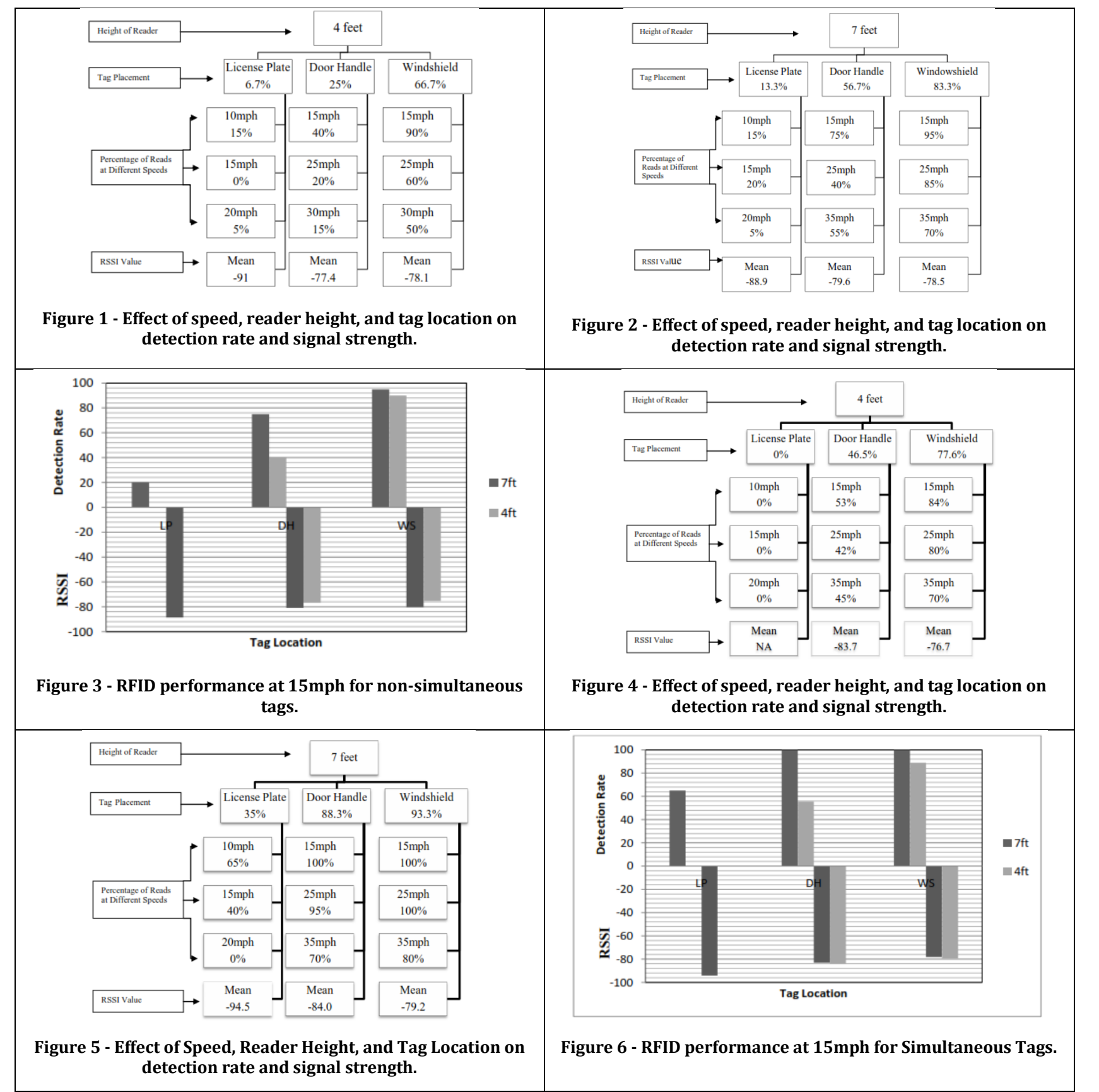

\section{Regression Analysis}

The regression analysis was performed to statistically quantify the effect of speed, tag location, and reader height on tag readability. Further, as observed in the descriptive analysis presented above, having multiple tags on the vehicle had an observable effect on tag readability, thus simultaneous tags versus a non-simultaneous variable was added in the analysis to quantify its effect. This variable was coded as a dummy with 1 if simultaneous and 0 otherwise. Similarly, to quantify the effect of horizontal distance between the tag and the reader, a dummy variable indicating lane number was included in the model, which takes a value of 1 for the shoulder lane that is close to the reader and 0 otherwise. The dependent variable was coded as binary taking a value of 1 if the tag was detected and 0 otherwise for each run. The results of logistic regression analysis are presented in Table 1. 
From Table 1 it is evident that all the variables were statistically significant at 5 percent significance level. Of all the variables, speed had negative impact on tag readability. Exponentiating the speed coefficient yields that for every unit increases in speed the tag readability decreases by 9.2 percent. This captures the effect of time lag between signals from the tag and reader. Further, the results show that a unit increase in reader height and tag height increases the probability of readability by 175.8 and 294.2 percent respectively. Horizontal distance has impact on tag readability. The inner/shoulder lane had 153.8 percent chance of being read compared to the outer lane, which was far from the reader location. This is in line with the expectations, the closer the tag is to the reader the better is the tag readability. Further, it was observed from Table 1 that the magnitude of the constant term was larger compared to variable coefficients. This implies that there are significant variables that are not included in the model.

Table 1 - Readability logit model results.

\begin{tabular}{|l|r|r|r|}
\hline Variable & Coefficient & Z-Statistic & P-Value \\
\hline Reader Height (ft) & 0.5644 & 7.77 & 0 \\
\hline Tag Height (ft) & 1.0790 & 13.82 & 0 \\
\hline Vehicle Speed (mph) & -0.0854 & -5.77 & 0 \\
\hline Lane Number (dummy) & 0.4302 & 2.16 & 0.03 \\
\hline Simultaneous Tags (dummy) & 1.4495 & 6.66 & 0 \\
\hline Constant & -5.8069 & -9.73 & 0 \\
\hline Pseudo R2 & & 0.3825 \\
\hline Pearson chi2(66) & \multicolumn{3}{|c|}{0.07 .67} \\
\hline Prob > chi2 & \multicolumn{3}{|}{0699} \\
\hline
\end{tabular}

\section{Conclusion}

This aim of this study was to investigate the effect of speed, tag and reader location, vehicle speed, and over-shadowing on RFID performance. The study tested two reader/antenna heights and three tag locations. Three vehicle speeds were tested for each tag location including the simultaneous location. Based on the results presented in this study, the following conclusions can be drawn: (1) Having tags individually placed on the vehicle clearly show that the optimal position for a tag on a vehicle with the given parameters was having the reader/antenna set at $7 \mathrm{ft}$ above the ground on a post and having the tag placed on the windshield. These tag/reader locations had the highest detection rates and signal strength. (2) Placing multiple tags on the vehicle simultaneously improved the signal strength and therefore, higher detection rates. It was expected that having multiple tags in close proximity would cause a shadow affect that would weaken the signal strength due to interference. In contrast, the highest detection rate was when the tag was placed at 7 feet and having multiple tags on the vehicle and the tag being placed on the window shield. (3) Vehicle speed has negative effect on tag detection rate. As shown in Table 1 and Figure 5, at higher vehicle speeds the detection rate decreases. (4) With respect to horizontal distance between the reader and the tag, the results show that the closer the tag and reader are to each other the higher the signal strength and so is the detection rate.

Recommendations for future studies are to try higher reader/antenna placement as well as higher speeds to investigate if higher detection rates can be achieved. Testing took several days due to time constraints and weather. Even though weather did not seem to have any effect on the readability, it would be better to have a controlled testing environment.

Furthermore, this research had a tag attached to a grass material on the windshield and metal material for the door handle and license plate and a reader attached to a metal traffic sign. This limits the conclusions to the conditions tested. Thus, for more general conclusions, it is recommended to carry out several tests that will account for different materials that are likely to be encountered in the real world in addition to what was covered in this research.

\section{References}

RF Code (2012), www.rfcode.com, Accessed March 29, 2012.

Stratos, (2011), http://www.stratos.com/projects/dero-zap. Accessed June, 2011.

Mwape, J.M., (2009), "Guide to Electronic Toll Payments", Xulon Press. The basics of RFID technology. http://www.rfidjournal.com/article/view/1337. Accessed July, 2011.

Houston Transtar, (2011), “Greater Houston Transportation and Emergency Management Center", www.houstonstanstar.org, accessed, June, 2011.

OOCEA, 2011, http://epass.oocea.com/Corporate/, Accessed July, 2011.

Fedrowitz, W. J., (2007), Implementation of RFID Technology in VT-VDOT Highway Maintenance Monitoring Program (HMMP). Master of Science Thesis, Virginia Polytechnic Institute and State University, Virginia.

Yates, N. J., (2009), Furthered Implementation of Long-Range RFID Technology in Highway Asset Management. Master of Science Thesis, Virginia Polytechnic Institute and State University, Virginia.

Mwape, J.M., (2009), "Guide to Electronic Toll Payments”, Xulon Press.

North Texas Toll Authority (NTTA), http://www.ntta.org, visited May 16, 2011.

Lodgher, A., Perkins, J., Yang, Y., Jones, E., and Hawkins, G., (2009), Feasibility and Applications of RFID Technologies to Support Right-OfWay Functions, Technical Report No. 0-6142-1, Texas Department of Transportation, Austin, Texas. 